Rechtsmedizin 2022 $\cdot 32: 282-288$ https://doi.org/10.1007/s00194-021-00537-x Angenommen: 8. September 2021 Online publiziert: 19. Oktober 2021 (c) Der/die Autor(en) 2021



\section{Die Abgrenzung der Fremdtötung von der eigenverantwortlichen Selbsttötung: Doppelsuizid, erweiterter Suizid oder Homizid- Suizid?}

\author{
E. Dietz · J. Schädler · K. Püschel · B. Ondruschka \\ Institut für Rechtsmedizin Hamburg, Universitätsklinikum Hamburg-Eppendorf, Hamburg, Deutschland
}

\title{
Zusammenfassung
}

In der Literatur finden sich vielfach Publikationen und Kasuistiken, die sich mit zeitlich, örtlich, partnerschaftlich und/oder familiär im Zusammenhang stehenden nichtnatürlichen Todesfälle unabhängig von der Art und Weise zweier oder mehrerer Personen auseinandersetzen. Im Nachfolgenden sollen jene Fälle genauer betrachtet werden, bei denen zumindest in einem der Fälle naheliegt, dass es sich beispielsweise aufgrund der Spurenlage um einen freiverantwortlichen Suizidentschluss gehandelt hat. Diese werden u. a. als „erweiterte Suizide“ oder in jüngerer Zeit auch als „HomizidSuizide" bezeichnet. Diese theoriespezifizierten Nomenklaturen finden sich nicht zuletzt deshalb berechtigt kritisiert, als dass die Motive, genauen Tatumstände und Tathintergründe oftmals nicht bekannt sind und somit eine (vorgreifende) Wertung erfolgt, ohne dass andere mögliche Tathergänge zur Diskussion gestanden hätten. Daher stellt sich zunächst die Frage nach der generellen und konkreten Eignung dieser Nomenklaturen hinsichtlich ihrer Anwendbarkeit, mit der sich daran anschließenden Überlegung einer neu zu definierenden übergeordneten Bezeichnung. Die Komplexität dieser Begrifflichkeiten soll im Nachfolgenden an dem gemeinsamen Tod eines älteren Ehepaares verdeutlicht werden.

\section{Schlüsselwörter}

Typologie · Schussverletzung · Paktsuizid · Gemeinschaftliche Selbsttötung · Postaggressioneller Suizid
Die Autoren E. Dietz und J. Schädler haben zu gleichen Teilen zum Manuskript beigetragen.

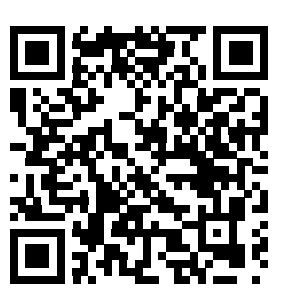

QR-Code scannen \& Beitrag online lesen

\section{Einleitung}

Fälle von Tötungen des Intimpartners, der ganzen Familie oder innerhalb verwandtschaftlicher und nichtverwandtschaftlicher Beziehungen mit anschließenden Selbsttötungen (Suizid) werfen die immer wiederkehrende Frage zur korrekten Benennung bzw. systematischen Nomenklatur solcher Taten auf. Der Begriff "Suizid" leitet sich aus dem Lateinischen "sui caedes" ab ("sui" = sich selbst und "caedere" = töten) und wird im deutschen Sprachgebrauch mit Selbsttötung, Selbst- mord und Freitod übersetzt und impliziert die bewusste und freiwillige Selbstschädigung (unabhängig von der Art und Weise) mit tödlichem Ausgang [1, 2]. Unter einem "Doppelsuizid" wird die freiwillige und jeweils selbst herbeigeführte Selbsttötung zweier Personen, vorrangig zum selben Zeitpunkt, am gleichen Ort sowie in gegenseitigem Einverständnis verstanden (Übersicht, Klassifikation der Suizidarten: - Tab. 1; [3]). Demgegenüber steht u. a. der Begriff des „erweiterten Suizides", bei welchem eine Person vor ihrer Selbsttötung auch einer anderen Person, 
Tab. 1 Begriffsdefinitionen/Begriffsverwendungen von Suizidalität. (Verändert und ergänzend adaptiert nach Wolfersdorf und Etzersdorfer [36])

\begin{tabular}{|c|c|}
\hline Nomenklatur & Bedeutung \\
\hline $\begin{array}{l}\text { (Einzel-)Suizid } \\
\text { (Selbsttötung, } \\
\text { Selbstmord, Frei- } \\
\text { tod) }\end{array}$ & $\begin{array}{l}\text { Bewusste und selbst herbeigeführte Handlung, mit der das eigene Le- } \\
\text { ben freiwillig beendet wird }\end{array}$ \\
\hline \multirow[t]{2}{*}{ Doppelsuizid } & $\begin{array}{l}\text { Freiwillige und jeweils selbst, d. h. durch die eigene Hand herbeigeführte } \\
\text { Selbsttötung zweier Personen }\end{array}$ \\
\hline & $\begin{array}{l}\text { Primär gleichzeitig, am gleichen Ort und im gegenseitigen Einverständ- } \\
\text { nis }\end{array}$ \\
\hline Paktsuizid & $\begin{array}{l}\text { Gemeinschaftlich beschlossener Plan zwischen } 2 \text { oder mehreren Perso- } \\
\text { nen zum Selbstmord. Geplanter bzw. verabredeter gemeinschaftlicher } \\
\text { oder getrennt ausgeführter, zeitlich terminierter Selbstmord }\end{array}$ \\
\hline \multirow{5}{*}{$\begin{array}{l}\text { Erweiterter Suizid } \\
\text { (Mitnahmesuizid, } \\
\text { „extended suicide“) }\end{array}$} & $\begin{array}{l}\text { Selbstmord nach vorangegangener Tötung mindestens einer anderen } \\
\text { Person, ohne deren Zustimmung und Wissen }\end{array}$ \\
\hline & $\begin{array}{l}\text { Freiwilligkeit des Todes des Mitgenommen wird impliziert, bleibt je- } \\
\text { doch offen (allgemeiner: Einverständnis zum Sterben ist nicht bei allen } \\
\text { Beteiligten vorhanden oder nicht eindeutig vorhanden) }\end{array}$ \\
\hline & $\begin{array}{l}\text { Die Mitnahme/Tötung eines anderen Menschen erwächst sekundär aus } \\
\text { der bestimmenden Selbsttötungsabsicht des Ausführenden }\end{array}$ \\
\hline & $\begin{array}{l}\text { Häufig Mitnahme geliebter Personen oder Personen für die man die } \\
\text { Verantwortung trägt, wie beispielsweise des Ehe- oder Liebespartners } \\
\text { (Intimizid) oder des Kindes (Infantizid, Filizid) }\end{array}$ \\
\hline & Psychodynamische Bedeutung der Beziehung \\
\hline \multirow{3}{*}{$\begin{array}{l}\text { Postaggressioneller } \\
\text { Suizid }\end{array}$} & Mord/Totschlag mit anschließendem Selbstmord \\
\hline & $\begin{array}{l}\text { Der Tötung einer anderen Person als Ausgangspunkt, schließt sich der } \\
\text { Selbstmord als mittelbare Reaktion an }\end{array}$ \\
\hline & Primär fremd-aggressiv \\
\hline \multirow[t]{4}{*}{$\begin{array}{l}\text { Homizid-Suizid } \\
\text { („,murder-suicide") }\end{array}$} & $\begin{array}{l}\text { Einbeziehung der Tötung/Ermordung eines (oder mehr) Menschen in } \\
\text { den eigenen Suizid }\end{array}$ \\
\hline & Unfreiwillige Mitnahme in den Tod \\
\hline & $\begin{array}{l}\text { Vorbeziehung zwischen Opfer und Täter kann fehlen/willkürliche Aus- } \\
\text { wahl der Opfer }\end{array}$ \\
\hline & Psychodynamisch oft Aspekte von Rache oder psychotische Vorgänge \\
\hline \multirow[t]{3}{*}{ Massensuizid } & Eigene Selbsttötung nach Tötung anderer \\
\hline & Mischung aus Freiwilligkeit, Zwang und Getötetwerden \\
\hline & Kombination von Homizid und Suizid \\
\hline \multirow[t]{4}{*}{ Amok } & Primäres Ziel ist die Tötung anderer \\
\hline & $\begin{array}{l}\text { Eigener Tod wird billigend in Kauf genommen, Selbsttötung im An- } \\
\text { schluss an die Tat häufig }\end{array}$ \\
\hline & U. a. als Extremum des Homizid-Suizids zu bezeichnen \\
\hline & Psychodynamisch eher fremd-aggressiv und paranoianahe \\
\hline \multirow[t]{3}{*}{$\begin{array}{l}\text { Suizidassoziierte } \\
\text { Todesfälle }\end{array}$} & $\begin{array}{l}\text { Jede Art von Todesfall, die im Zusammenhang mit einer Selbsttötung } \\
\text { steht, ohne initiale Wertung/Deutung der möglichen Beweggründe }\end{array}$ \\
\hline & Greift einer vorzeitigen Täter- und Opfermachung vor \\
\hline & tur, im Sinne einer Überbegrifflichkeit \\
\hline
\end{tabular}

ohne deren Zustimmung oder deren Wissen, jedoch unter deren angenommenen bzw. unterstellten Freiwilligkeit (autorenabhängig) das Leben nimmt [4-8]. Hiervon abzugrenzen sind wiederum der "postaggressionelle Suizid" und der "homicide- bzw. murder-suicide", bei denen die Freiwilligkeit des Getötetwerdens auf der Opferseite fehlt [9]. Beispiele, Ka- letztlich ebenso wie die Abgrenzung zu einem Tötungsdelikt mit anschließendem Suizid bzw. vorgetäuschtem Suizid nur durch eine interdisziplinäre Zusammenarbeit zwischen Ermittlungsbehörde und Rechtsmedizin voneinander abgegrenzt werden [14-17, 20]. Die möglichen Interpretationsspielräume sowie die unterschiedlichen Begrifflichkeiten im Zusammenhang mit der eigenbestimmten Selbsttötung sollen im Nachfolgenden anhand eines Fallbeispiels erläutert werden.

\section{Kasuistik}

Sachverhalt. Ein Rentnerehepaar wurde in seiner Wohnung leblos im Schlafzimmer, auf dem Bett liegend, durch Angehörige aufgefunden (0 Abb. 1). Die Polizei wurde verständigt.

Auffindesituation. Auf der linken Betthälfte lag in Linksseitenlage ein weiblicher Leichnam mit mittels Bettdecke abgedecktem Kopf. Auf der rechten Betthälfte befand sich ein in Rückenlage liegender, männlicher Leichnam, der in seiner rechten Hand eine Pistole hielt, welche auf dem Rumpf lag. Beide Verstorbenen waren regelrecht bekleidet und wiesen jeweils für einen Schuss typische Verletzungen im Bereich der rechten Schläfen-/ Scheitelbeinregionen auf. Das Kopfhaar der Frau war, insbesondere linksseitig, diffus blutig verklebt. Blutabrinnspuren waren nicht abgrenzbar. Der Mann wies eine zusätzliche Kopfschwartenverletzung an der linken Kopfseite auf. Beidseits von den Verletzungen nach hinterhauptwärts verliefen in querer Richtung angetrocknete Blutabrinnspuren. Es waren grobsichtig keine weiteren Verletzungen erkenntlich, die auf ein unmittelbares Kampfgeschehen hindeuteten. Zudem fanden sich augenscheinlich keine abgrenzbaren spritzerartigen Blut- und Gewebsantragungen im Sinne sog. Rückschleuderspuren aus den Einschusswunden an den Handrücken beider Verstorbener. Ebenso fehlten Beschmauchungen (grau-schwärzliche Pulverschmauchablagerungen) an den Händen beider Personen.

Fremdanamnese. Der verstorbene Mann sei mutmaßlich depressiv gewesen und habe vor mehreren Jahren einmalig Selbst- 


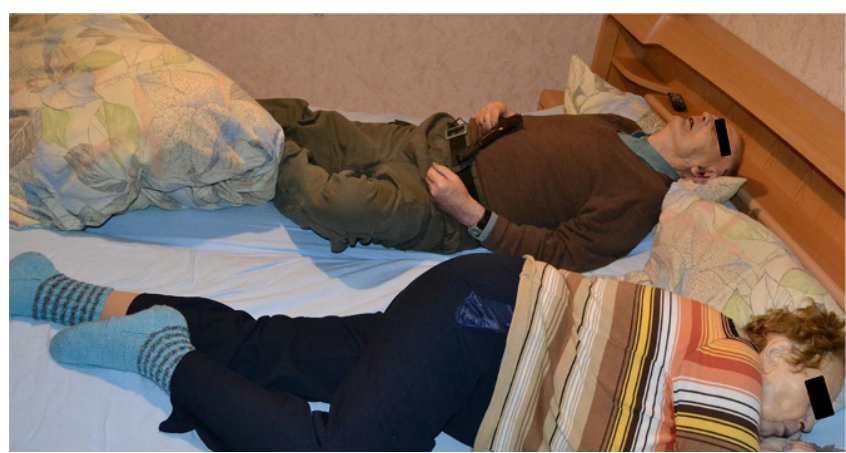

Abb. $1 \triangleleft$ Auffindesituation des im Bett tot aufgefundenen und durch Kopfschüsse ums Leben gekommenen Ehepaares
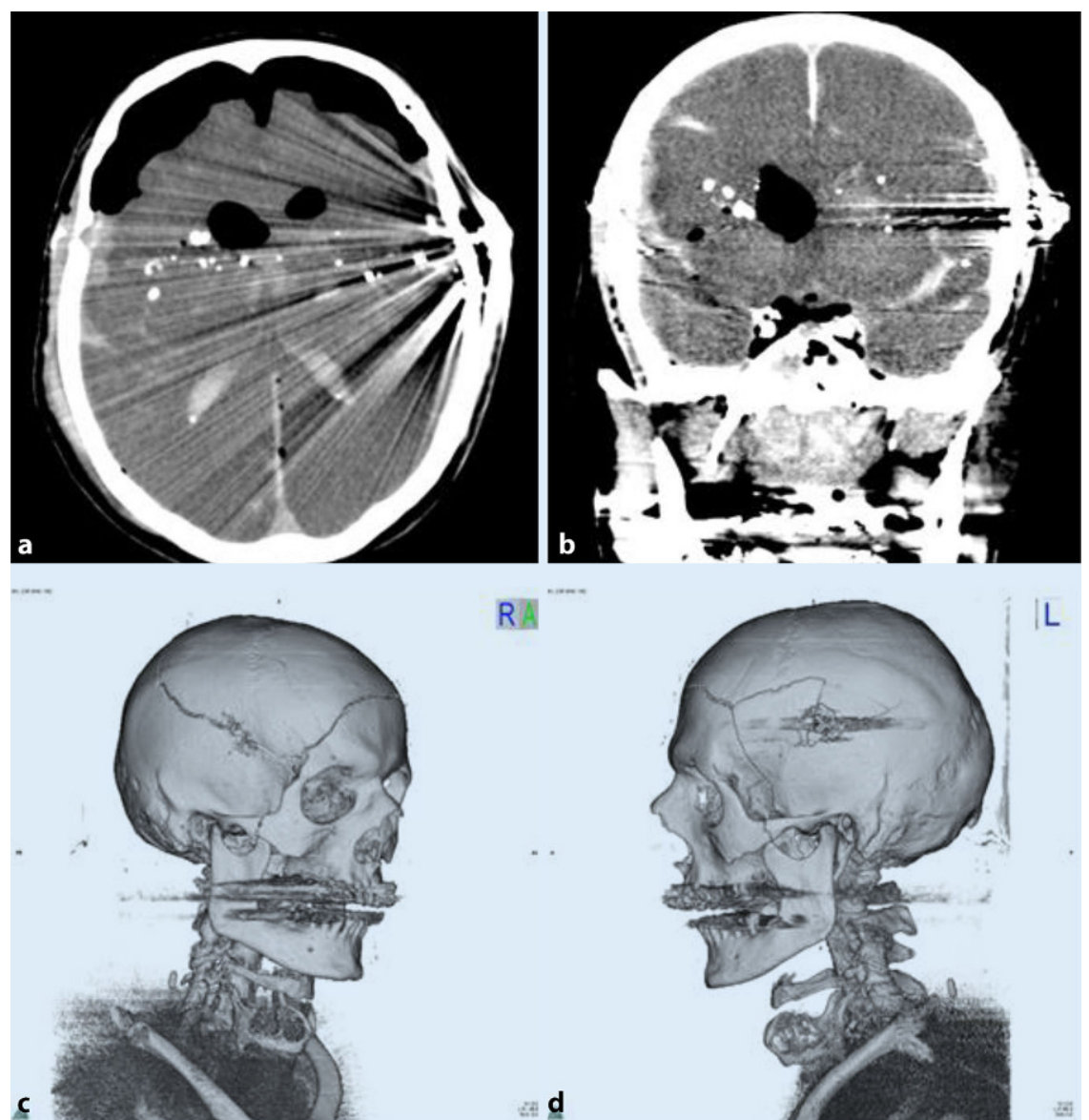

Abb. 2 ム Postmortale CT-Aufnahmen des Ehemannes. Nahezu horizontaler "Durchschuss" durch die Großhirnhemisphären in planer Ebene. Einschuss rechtsseitig mit Ausschuss/Steckschuss linksseitig. Um den Einschuss- und Steckschussdefekt Frakturlinien und Brüche. Steckendes, deformiertes Projektil linksseitig zwischen Schädelknochen und Kopfschwarte und den Schusskanal säumende Projektilfragmente: $\mathbf{a}$ axiale Schnittebene, $\mathbf{b}$ koronare Schnittebene, $\mathbf{c}, \mathbf{d}$ 3D-Rekonstruktion

mordabsichten unter Mitnahme seiner Frau gegenüber seinem Bruder geäußert. Starke psychische Schwankungen oder emotionale Belastungen seien in den letzten Jahren nicht bekannt geworden. Seine Frau sei alters- und erkrankungsbedingt, ersten Ermittlungsergebnissen zufolge, etwas bewegungseingeschränkt gewesen. Wesentliche schwere Grundund Begleiterkrankungen, insbesondere solche bei denen mit schweren Verläufen und aggressiven Therapien zur Behandlung zu rechnen gewesen wäre, seien bei beiden nicht bekannt gewesen. Neuerliche Ankündigungen von Selbstmordabsichten oder ein Abschiedsbrief hätten nicht vorgelegen. Ferner habe in Erfahrung gebracht werden können, dass der Ehemann Sportschütze gewesen sei und legal Schusswaffen besessen habe.
Äußere und innere Leichenschau. Bei beiden Verstorbenen fanden sich äußerlich keine Zeichen einer stumpfen Gewalteinwirkung als Hinweise auf ein stattgehabtes Kampfgeschehen. Morphologisch wurden keine Rückschleuderspuren, Beschmauchungen oder Schlittenverletzungen an den Händen beider Personen festgestellt. Kriminaltechnisch gelang in weiterführenden Untersuchungen ein Nachweis von geringen Schmauchrückständen an der rechten Hand des Mannes. Im Rahmen der gerichtlichen Sektion konnten bei beiden Leichnamen altersentsprechende Veränderungen des Organ- und Gefäßsystems, ohne schwere akute oder chronische, den Tod unmittelbar determinierende Erkrankungen nachgewiesen werden. In beiden Fällen fanden sich die klassischen Zeichen eines absoluten bzw. eines näheren relativen Nahschusses mit Verbleib der Geschosse im Schädel (Steckschüsse; - Abb. 2a-d und 3a-d).

Problemidentifikation. Die i. Allg. bei Schusswaffengewalt zur Differenzierung von Suiziden und Fremdtötungen herangezogenen Befunde, wie schussassoziierte Blutspuren (Rückwärtsspritzer entgegen der Flugrichtung des Geschosses/Projektils auf der den Schuss abgebenden Hand bei geringer Schussdistanz), Beschmauchungen (Pulverschmauch an den Händen/der Hand des Schützen), Verbrennungen (durch glühende Pulverpartikel), Schlittenverletzungen (Hautabschürfungen oder Unterblutungen durch die Kanten des zurückgleitenden Verschlussstückes einer Pistole), Zeichen der äußeren Gewalteinwirkung als Hinweise eines möglichen vorgelagerten Kampfgeschehens oder eine Ankündigung der geplanten Tat gegenüber Dritten bzw. eine Erklärung der vollzogenen Tat (Abschiedsbrief) sowie Hinweise auf das Vorliegen von starken sozialen, wirtschaftlichen und/oder gesundheitlichen Problemen, fanden sich im gegenständlichen Fall nicht.

Mehr oder weniger suizidtypisch zeigten sich lediglich die gewählten Einschusslokalisationen, d. h. Abzielung auf lebenswichtige, den Tod schnell und sicher herbeiführende Organ(anteil)e, die nach den Befunden geringe Schussentfernung (aufgesetzter Schuss bzw. absoluter Nahschuss bei Suiziden häufiger) sowie die in der 

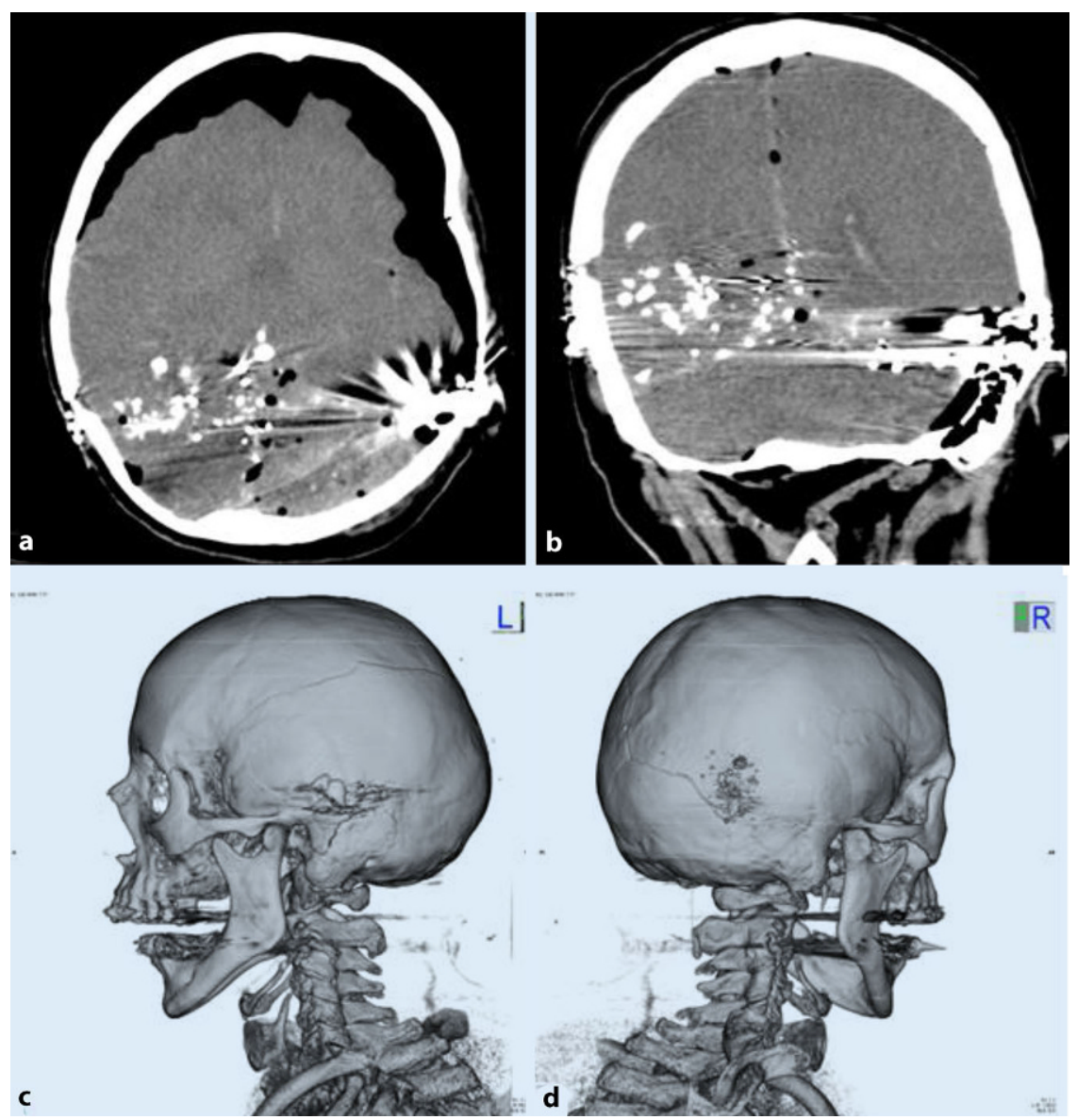

Abb. 3 ॥ PostmortaleCT-Aufnahmen derEhefrau.LeichtschrägerDurchschuss durch diehinteren Teile der Großhirnhemisphären unter Einbeziehung des Hirnstamms und angrenzender Kleinhirnanteile. Einschuss rechtsseitig mit Steckschuss linksseitig in der seitlichen Schädelbasis (Schläfenbeinpyramide). Um den Einschuss- und Steckschussdefekt Frakturlinien und Brüche. Um den Schusskanal herum säumende Projektilfragmente. a Axiale Schnittebene, b koronare Schnittebene, c, d 3D-Rekonstruktion

Hand gehaltene Waffe im Falle des Mannes mit Nachweis von geringen Schmauchantragungen. Zudem war der Mann Sportschütze und somit im legalen Besitz von Schusswaffen mit hierdurch erleichtertem Zugang zu einem Tatwerkzeug.

Sonach ergeben sich zum Sektionszeitpunkt u.a. folgende mögliche und denkbare Tathergänge:

1. Doppelsuizid - Das Ehepaar entschloss sich gemeinschaftlich zum Selbstmord. Nachdem sich die Frau mittels eigenhändiger Schussabgabe suizidiert hatte, nahm der Ehemann die Pistole an sich und suizidierte sich ebenfalls.

2. Tötung auf Verlangen mit anschlieBendem Suizid - Das Ehepaar entschloss sich gemeinschaftlich zum Selbstmord. Da sich die Frau nicht selbst suizidieren wollte, vereinbarte das Ehepaar, dass der Mann erst sie und im Anschluss sich selbst suizidieren solle.

3. Beihilfe zum Suizid mit anschließendem Suizid - Die Ehefrau entschloss sich zum Suizid und bat ihren Mann (Sportschütze), ihr eine Pistole für die angestrebte Tat bereitzustellen. Nachdem sich die Frau mittels Kopfschuss suizidierte, plagten den Mann Schuldgefühle, sodass er sich entschloss, sich ebenfalls zu suizidieren.

4. Postaggressioneller Suizid - Der Ehemann sah sich zunehmend mit der Unterstützung seiner Ehefrau im Alltag überfordert und entwickelte Aggressionen, die sich in einer Tötung seiner Frau externalisierten. Im Anschluss fand dieser keinen Ausweg und suizidierte sich.

5. Erweiterter Suizid - Der Ehemann entschloss sich aufgrund einer psychi- schen Störung bzw. psychiatrischen Erkrankung, z. B. im Rahmen einer schweren depressiven Episode, zum eigenen Selbstmord und erschoss seine Ehefrau - ohne Einholung der Zustimmung und Möglichkeit zur freien Willensentscheidung, jedoch unter Zugrundelegung der implizierten Freiwilligkeit, bevor er sich selbst erschoss.

6. Tötungsdelikt mit Anschlusssuizid Im Rahmen einer psychiatrischen Erkrankung bzw. einer akuten Psychose, beispielsweise vermittelt durch Wahnvorstellungen oder Halluzinationen, erschoss der Ehemann seine Frau, wurde sich im Nachgang dessen bewusst, und suizidierte sich.

Alle angeführten Beispiele, liegen mit gröBerer oder geringerer Wahrscheinlichkeit - zumindest nach den Sektionsergebnissen und den vorläufigen Ermittlungsergebnissen - im Bereich des Möglichen. Die nachgewiesenen Schmauchantragungen an der rechten Hand des Mannes bei fehlendem Schmauchnachweis an den Händen der Frau lassen vermuten, dass der Mann der Schütze war, sodass die Varianten 1 und 3 eher unwahrscheinlich erscheinen. Eine (angenommene) Einvernehmlichkeit seitens der Frau zur Tötung ist damit aber nicht bewiesen bzw. eine Selbsttötung der Frau nicht gänzlich ausgeschlossen.

\section{Diskussion}

Im Hinblick auf die Anwendbarkeit der einleitend angeführten Nomenklaturen zur Kategorisierung von gemeinschaftlichen Selbsttötungen oder von Selbsttötungen nach vorangegangener Tötung mindestens einer anderen Person bedeutet dies, dass die genauen Umstände, unter denen der Tod eintrat bzw. unter denen die Tötung eines anderen Menschen vollzogen wurde, zweifelsfrei und ohne Interpretationsspielraum bekannt sein müssen. Hierzu kann neben einer ",psychologischen Autopsie" durch Befragung von Angehörigen bzw. nahestehenden Personen zur Erkundung der Beweggründe des Suizidenten auch eine "social media autopsy", d.h. die Auswertung von sozialen Medien oder Web-Accounts (Facebook, Insta- 
gram etc.) hilfreich sein. Gerade bei Suizidfällen mehrerer Personen fällt jedoch diese Möglichkeit der Informationsbeschaffung naher Angehöriger weg, da diese sich ggf. selbst mitgetötet haben bzw. wurden [21, 22, 24, 25]. Die genauen Todesumstände sind nur in den wenigsten Fällen tatsächlich vollständig bekannt, weshalb die Anwendbarkeit solcher theoretischer Nomenklaturen in der Praxis häufig schwierig ist $[23,24]$. Eine begriffliche Klassifizierung des erweiterten Suizids kann Opfern grundsätzlich nicht gerecht werden: Sie ist täter- und nicht opferorientiert. Dem erweiterten Suizid kann der Umstand zugeschrieben werden, dass die Tötung eines anderen Menschen als Handlungseinheit sekundär aus der Verwirklichung der im Vordergrund stehenden und intendierten Selbsttötung erwächst $[8,25]$. Dies bedeutet, dass sich die externalisierenden negativen und zu Selbst- und Fremdtötung führenden Gefühle primär gegen das Selbst des Tötenden und nur sekundär gegen den Getöteten richten.

Demgegenüber steht u.a. der postaggressionelle Suizid, der Mord oder Totschlag mit anschließender Selbsttötung. Hierbei steht die Tötung eines anderen Menschen im Vordergrund (z. B. Ehe- oder Eifersuchtsmord), der schließlich die Selbsttötung als letztmögliche Handlung bzw. als Reaktion auf eine sich daraus ergebende aussichtslose Lage (beispielsweise drohende Anklage oder Inhaftierung, quälende Schuldgefühle) folgt $[3,25,26]$. Auch kombinierte Formen sind möglich. Beispielhaft zu erwähnen wäre der Ehefrauenmord aus Eifersucht, wobei sich der Ehemann erst nach der Tötung seiner Frau der Tat bewusst wird und vor der Selbsttötung auch die gemeinsamen Kinder tötet, damit diese nicht alleine und ohne Eltern zurückbleiben.

Etwas weiter gefasst zeigen sich die beiden Nomenklaturen des Homizid-Suizids („homicide-suicide“) und des Mord-Suizids („,murder-suicide“), wobei ersterer Begriff am wenigsten direktiv, jedoch nicht gänzlich wertneutral ist. So impliziert "homicide" ein Tötungs- und "murder" ein Mordgeschehen [27]. Der eigene Suizid der ausführenden Person darf dabei definitionsgemäß bis zu einer Woche nach der Tötung einer anderen Person stattfinden [9, 10]. Nach unterschiedlichen Studien sind bis zu 90\% der Täter männlich und töten gehäuft zunächst ihre Lebenspartnerinnen/ Intimbeziehungen und anschließend sich selbst, bevorzugt mit Schusswaffen. Frauen töten bevorzugt zunächst ihre Kinder oder aber ihren Ehemann, wobei in diesen Fällen häufig ein sexueller Missbrauch vorausgegangen ist, bevor sie sich selbst suizidieren [9-13]. Um beispielsweise einer vorzeitigen Tätermachung vorzubeugen, und um ein Tatgeschehen folgerichtig erfassen zu können, ist die Anwendbarkeit dieser Nomenklaturen also folglich nur dann gegeben, wenn auch letzte geringe Zweifel, auch im Hinblick des theoretisch Möglichen, ausgeräumt werden können, s. das hier vorgestellte Beispiel. Nur bei einer der beiden Personen ergaben sich anhand der Spurenlage und -beschaffenheit am Tatort eindeutige Hinweise auf einen Suizid, wohingegen bei der anderen Person sowohl eine Selbst- als auch eine Fremdtötung in Betracht gezogen werden können. Auch durch die polizeilichen Ermittlungen ließ sich abschließend eine Fremdtötung im vorgestellten Fall nicht mit an Sicherheit grenzender Wahrscheinlichkeit ausschließen. Die Obduktionsergebnisse wiesen keine Verletzungen nach, die auf ein Kampfgeschehen hindeuteten, sodass dieses möglicherweise als Indiz gewertet werden kann, dass die Tötung der Frau - sofern diese nicht durch sie selbst erfolgte - einvernehmlich stattfand.

Auch beim Doppelsuizid scheinen sich die Kriterien der begrifflichen Definition nur selten und ohne Deutungsoffenheit zu bestätigen $[9,10]$. Dies ist dann der Fall, wenn ein Tötungsmittel gewählt wird, welches auf zwei Personen gleichzeitig einwirkt $[18,19,25]$. Zu nennen wären hierbei das Trinken einer mit Gift versetzten Flüssigkeit (beispielsweise in Wein aufgelöstes Zyankali oder Rizin), das Aufstellen von Holzkohlegrills in geschlossenen Räumen, das Lenken eines Kraftfahrzeuges gegen ein Hindernis und das gemeinschaftliche Herabstürzen aus großer Höhe. Das Problem des Begriffs besteht in der Tatsache, dass letztlich offenbleibt, ob es sich um eine frei gewählte Handlung beider handelte. Zwar können Tatankündigungen in mündlicher (gegenüber Dritten geäußerte Vorhaben und Gedanken) oder schriftlicher Form (Abschiedsbrief) Hinweise auf das Tatgeschehen geben, letztlich werden aber immer wieder Fragen offenbleiben oder sich neue Fragen ergeben, wie beispielsweise: War es bis zuletzt der gemeinschaftliche Wille? Wusste der Mitgetötete um die unmittelbar bevorstehende Planausführung und bestand die Möglichkeit der freien Willensumentscheidung?

Ähnlich dem Doppelsuizid, jedoch eindeutiger im Hinblick auf die freie Willensentscheidung zur Lebensbeendigung, verhält es sich beim sog. Paktsuizid („,suicide pact ${ }^{\prime \prime}$ ). Hierbei handelt es sich definitionsgemäß um eine terminierte Verabredung zum Selbstmord, beispielsweise in elektronischer Form per Telefon, E-Mail, Internetforen etc. Dieser "Pakt " kann gemeinschaftlich oder getrennt voneinander erfolgen. Letzteres legt die freie Willensentscheidung nahe, jedoch bleibt die Möglichkeit einer bewussten Ausnutzung emotionaler Abhängigkeiten oder der Erpressung bestehen [28-32].

Autorenabhängige Versuche der (Neu-/Re-)Interpretation der bestehenden Nomenklaturen mit Vereinfachungen, Verallgemeinerungen und Zusammenfassungen, teilweise einschließlich der synonymen Verwendung der unterschiedlichen Nomenklaturen, gestalten sich problematisch. Neue Definitionen bzw. Auslegungen zeigen ebenfalls kritisierbare Ansätze hinsichtlich Ein- und Ausschlusskriterien auf. Erwähnt wäre exemplarisch der erweiterte Suizid, beispielsweise definiert als die Einbeziehung eines oder mehrerer Menschen ohne deren Einverständnis oder gegen deren Willen in eine Tötungshandlung durch eine zur Selbsttötung entschlossene Person, u.a. unter Ausschluss von postaggressionellen Suiziden und von Suiziden nach vorangegangener einvernehmlicher Partnertötung [33, 34].

Die üblichen Definitionen sind in vielen Fällen nicht weit genug gefasst und nicht wertfrei. Dahingehend bleiben insbesondere zu Beginn einer Todesermittlung, aber auch nach deren Abschluss einzelne Ermittlungsfragen oft ungeklärt. Die Zusammenhänge von Mord und Selbstmord stellen in der Vielzahl der Fälle ein nicht ganzheitlich erfassbares Geflecht von komplexen Interaktionsebenen dar [28, 29, $35,36]$. Es bedarf einer übergreifenden Bezeichnung, die gleichzeitig spezifisch genug gefasst ist, um Todesfälle, die einer 
Selbsttötung vor-oder nachgeschaltet und mit dieser in einen zeitlichen, örtlichen und situativen Zusammenhang zu bringen sind, benennen und eingruppieren zu können. Sie sind zudem nur sinnvoll, wenn sie zumindest potenziell eindeutig sind, wenig Spielraum für Interpretationen lassen und autorenunabhängig in gleicher Weise verwendet werden. Eine mögliche Umschreibung wäre der Begriff der suizidassoziierte Todesfälle („suicide-connected deaths" oder "suicide-connected death cases"); einer aus der englischsprachigen Literatur stammenden und zur Eingruppierung von anderen im Zusammenhang stehenden Todesfällen angewandten Bezeichnung (wie beispielsweise im Falle von "service-connected deaths" und „battleconnected deaths") [37].

\section{Fazit für die Praxis}

Die Klassifizierung bzw. Eingruppierung von im Zusammenhang stehenden Todesfällen entsprechend deren begrifflichen Abgrenzung - beispielsweise in "erweiterte Suizide", "Homizid-Suizide", ,postaggressionelle Suizide" und "Doppelsuizide" -, sollte möglichst erst nach Abschluss der Ermittlungen erfolgen, mit dem Ziel des Vorbeugens einer vorgreifenden Täter- und Opfermachung, auch im Hinblick auf den womöglich diffamierenden Charakter und der Gefahr einer vorwegnehmenden Wertung. Eine zwischenzeitliche mögliche Umschreibung wäre der Begriff der suizidassoziierten Todesfälle. Polizeiliche Ermittlungen sind notwendig, um in Verbindung stehende Todesfälle von Tötungsdelikten mit nachfolgender Selbsttötung des Täters sicher abzugrenzen und ein strafrechtlich relevantes Fremdverschulden einer dritten Person sicher ausschließen zu können. Außer der Tatortsicherung, Tatortuntersuchung unter Hinzuziehung eines Rechtsmediziners und der äußeren und inneren Leichenschau gehört hierzu eine genaue Umfeldanalyse, ergänzt um die Sozial- und Krankenanamnese. Die letztliche Bewertung eines Todesfalles als Tötung auf Verlangen, Beihilfe zum Selbstmord, Totschlag oder Mord ist Aufgabe von Staatsanwaltschaften und Gericht und weist eine große Praxisrelevanz auf, insbesondere im Hinblick auf Risikolebensversicherungen, Todesfallstatistiken und die für die trauernden Hinterbliebenen notwendige Aufklärungsarbeit.

\section{Korrespondenzadresse}

\section{Dr. med. E. Dietz}

Institut für Rechtsmedizin Hamburg, Universitätsklinikum Hamburg-Eppendorf Butenfeld 34, 22529 Hamburg, Deutschland e.dietz@uke.de

Funding. Open Access funding enabled and organized by Projekt DEAL.

\section{Einhaltung ethischer Richtlinien}

Interessenkonflikt. E. Dietz, J. Schädler, K. Püschel und B. Ondruschka geben an, dass kein Interessenkonflikt besteht.

Die Untersuchungen erfolgten unter Einhaltung der Vorgaben der Zentralen Ethikkommission der Bundesärztekammer.

Open Access. Dieser Artikel wird unter der Creative Commons Namensnennung 4.0 International Lizenz veröffentlicht, welche die Nutzung, Vervielfältigung, Bearbeitung, Verbreitung und Wiedergabe in jeglichem Medium und Format erlaubt, sofern Sie den/die ursprünglichen Autor(en) und die Quelle ordnungsgemäß nennen, einen Link zur Creative Commons Lizenz beifügen und angeben, ob Änderungen vorgenommen wurden.

Die in diesem Artikel enthaltenen Bilder und sonstiges Drittmaterial unterliegen ebenfalls der genannten Creative Commons Lizenz, sofern sich aus der Abbildungslegende nichts anderes ergibt. Sofern das betreffende Material nicht unter der genannten Creative Commons Lizenz steht und die betreffende Handlung nicht nach gesetzlichen Vorschriften erlaubt ist, ist für die oben aufgeführten Weiterverwendungen des Materials die Einwilligung des jeweiligen Rechteinhabers einzuholen.

Weitere Details zur Lizenz entnehmen Sie bitte der Lizenzinformation auf http://creativecommons.org/ licenses/by/4.0/deed.de.

\section{Literatur}

1. Neuner I, Schneider F (2016) Suizidalität. In: Schneider F (Hrsg) Klinikmanual: Psychiatrie, Psychosomatik und Psychotherapie, 2. Aufl. Springer, Heidelberg, Berlin, S 597-604

2. Otmar J (1986) Die Zuverlässigkeitsproblematik der Todesursachenstatistik, insbesondere der Rubrik "Selbstmord", deren Veränderung in der Vergangenheit und die Auswirkungen auf die Praxis. In: Haesler WT, Schuh J (Hrsg) Der Selbstmord/Le suicide. Rüegger, Grüsch, S83-103

3. Heuyer G (1973) Psychoses collectives et Suicides collectifs. PUF, Paris, S1-128

4. Näcke P (1908) Der Familienmord in gerichtlich-psychiatrischer Beziehung. Z Gerichtl Med 35:137-157

5. Bien S (1984) Der erweiterte Suizid. In: Faust V, Wolfersdorf M (Hrsg) Suizidgefahr: Häufigkeit - Ursachen - Motive - Prävention - Therapie. Hippokrates, Stuttgart, S31-39
6. Bien S (1986) Der erweiterte Suizid bei psychiatrischen Patienten. In: Pöldinger W, Reimer CH (Hrsg) Psychiatrische Aspekte suizidalen Verhaltens. Das Ärztliche Gespräch H37, S62-75

7. Witter H, Luthe R (1966) Die strafrechtliche Verantwortlichkeit beim erweiterten Suizid. Monatsschr Kriminol 49:97-113

8. Popella E (1964) Über den erweiterten Suizid. ZNeurPsych 205:615-624

9. Saint-Martin P, Bouyssy M, O'Byrne P (2008) Homicide-suicide in Tours, France (2000-2005)—description of 10 cases and a review of the literature. J Forensic Leg Med 15(2):104-109. https://doi.org/10.1016/j.jflm. 2007.03 .006

10. Milroy CM, Dratsas M, Ranson DL (1997) Homicidesuicide in Victoria, Australia. Am J Forensic Med Pathol 18(4):369-373. https://doi.org/10.1097/ 00000433-199712000-00011

11. Chan Y, Beh SL, Broadhurst RG (2003) Homicidesuicide in Hong Kong, 1989-1998. Forensic Sci Int 137(2-3):165-171. https://doi.org/10.1016/ S0379-0738(03)00350-5

12. Lecomte D, Fornes $P$ (1998) Homicide followed by suicide: paris and its suburbs, 1991-1996. J Forensic Sci 43(4):760-764. https://doi.org/10. 1520/JFS14303J

13. Milroy CM (1993) Homicide followed by suicide (dyadic death) in Yorkshire and Humberside. Med Sci Law 33(2):167-171. https://doi.org/10.1177/ 002580249303300213

14. Binder S, Banaschak S, Beike J, Rothschild MA, Kamphausen T (2018) Vom Rücken durch die Brust ins Auge - Ungewöhnlicher gemeinschaftlicher Suizid mittels einer Schusswaffe. Rechtsmedizin 28:423-428. https://doi.org/10.1007/s00194018-0248-4

15. Karger B, Brinkmann B (1997) Multiple gunshot suicides: potential for physical activity and medicolegal aspects. Int J Legal Med 110:188-192. https:// doi.org/10.1007/s004140050065

16. Karger B, Billeb E, Koops E, Brinkmann B (2002) Autopsy features relevant for discrimination between suicidal and homicidal gunshot injuries. Int J Legal Med 116(5):273-278. https://doi.org/ 10.1007/s00414-002-0325-8

17. Kamphausen T,AlthausL, KüpperU, RothschildMA, Bajanowski T (2014) Tödliche Schussverletzung eines Motorradrockers. Rechtsmedizin 24:502-507. https://doi.org/10.1007/s00194-014-0981-2

18. Faller-Marquardt M, Pollak S (2000) Gemeinschaftlicher Suizid mit Schussabgabe in die Scheitelregion. Rechtsmedizin 10:148-152. https://doi.org/ 10.1007/s001940000053

19. Dolenc A, Lovsin J (1980) Selbstmord zweier Personen. Beitr Gerichtl Med 38:53-356

20. KargerB (1996)ZurUnterscheidung von Jagdunfall und Suizid mit Langwaffen. Versicherungsmedizin 48(1):11-15

21. Aquila I, Sacco MA, Gratteri S, Sirianni M, De Fazio P, Ricci P (2018) The "social-mobile autopsy": the evolution of psychological autopsy with new technologies in forensic investigations on suicide. Leg Med 32:79-82. https://doi.org/10.1016/j. legalmed.2017.12.008

22. Isometsä ET (2001) Psychological autopsy studies-a review. Eur Psychiatry 16(7):379-385. https://doi.org/10.1016/S0924-9338(01)00594-6

23. Deschins J, Holz F, Duttge G et al (2020) Der Doppelsuizid - Teil 1: juristische Aspekte und historische/zeitgeschichtliche Fälle. Rechtsmedizin 30:438-450. https://doi.org/10.1007/s00194020-00433-w 
24. Holz F, Verhoff MA, Deschins J, Duttge G, Parzeller M (2021) Der Doppelsuizid - Teil 2: rechtsmedizinische und kriminalistische Aspekte. Rechtsmedizin 31:4249. https://doi.org/10.1007/ s00194-020-00434-9

25. Haenel T (2001) Definition der Begriffe „Doppelsuizid" und "erweiterter Suizid". In: Haenel T (Hrsg) Suizid und Zweierbeziehung. Vandenhoeck \&Ruprecht, Göttingen, S40-43

26. Warnke $A$ (2008) Suizid und Suizidversuch - Suizidalität. In: Herpertz-Dahlmann B, Resch F, SchulteMarkwort M, Warnke A (Hrsg) Entwicklungspsychiatrie: Biopsychologische Grundlagen und die Entwicklung psychischer Störungen. Schattauer, Stuttgart, S 1006-1007

27. Frei A (2014) Der Stellenwert der psychologischen Autopsieim Fallevon Homizid-Suizid.In:NiggliMA, Marty L (Hrsg) Risiken der Sicherheitsgesellschaft: Sicherheit, Risiko \& Kriminalpolitik. Forum, Mönchengladbach, S183-194

28. Foerster K (2009) „Erweiterter Suizid“: Ein problematischer Begriff? Nervenarzt 80:1078-1084

29. Rasch W (1979) Erscheinungsbild, Dynamik und Beurteilung des erweiterten Selbstmords. ZVersicherungswirtsch 68:417-426

30. Nock MK, Marzuk PM (2000) Suicide and violence. In: Hawton K, Heeringen K (Hrsg) The international handbook of suicide and attempted suicide. Wiley, Chichester, New York, S437-458

31. White T (2011) Working with suicidal individuals: a guide to providing understanding, assessment and support. Jessica Kingsley Publishers, London, Philadelphia, S64-67

32. Fishbain DA, D'Achille L, Barsky S et al (1984) A controlled study of suicide pacts. J Clin Psychiatry 45(4):154-157

33. Pollak S (1979) Erscheinungsformen des erweiterten Selbstmordes. Forensia 2:67-78

34. Pollak S (2005) Rechtsmedizinische Aspekte des Suizids. Rechtsmedizin 15:235-249

35. Hellen F, Lange-Asschenfeldt C, Huckenbeck W, Hartung B (2014) Der "erweiterte Suizid" Vollendete Homizid-Suizide unter psychopathologischen und kriminologischen Aspekten. Nervenarzt 85:1144-1150

36. Wolfersdorf M, Etzersdorfer E (2011) Begriffsbestimmung und Formen von Suizidalität. In: Wolfersdorf $M$, Etzersdorfer $E$ (Hrsg) Suizid und Suizidprävention. Kohlhammer, Stuttgart, S24-37

37. Maynard C, Trivedi R, Nelson K, Fihn SD (2018) Disability rating, age at death, and cause of death in U.S. Veterans with service-connected conditions. Mil Med 183(11-12):e371-e376. https://doi.org/ 10.1093/milmed/usy040

\section{The distinction between killing others and killing oneself: double suicide, extended suicide or homicide suicide?}

There are many publications and case histories, which describe non-natural deaths related to time, place, partnership and/or family, irrespective of the manner of two or more persons. We wanted to analyze cases in more detail, in which at least one case suggests a freely responsible suicide conclusion. In the literature they are described as extended suicides or more recently as homicide suicides. These theory-specific terms are justifiably criticized not least because the motives, exact circumstances and background of the crime often remain unknown. Therefore, the question of the general and concrete suitability of such terms arises, with the consideration of a new superordinate term to be defined. The complexity of these terms is illustrated in the following by the joint death of an older couple.

\section{Keywords}

Typology · Gunshot wound · Pact suicide · Joint suicide · Postaggressive suicide 